\title{
Geometric Phase Induced by a Cyclically Evolving Squeezed Vacuum Reservoir
}

\author{
Angelo Carollo, ${ }^{1,2}$ G. Massimo Palma, ${ }^{3,4}$ Artur Łozinski, ${ }^{3}$ Marcelo França Santos, ${ }^{5}$ and Vlatko Vedral ${ }^{6}$ \\ ${ }^{1}$ Centre for Quantum Computation, DAMTP, University of Cambridge, Wilberforce Road, Cambridge CB3 OWA, United Kingdom \\ ${ }^{2}$ Institute for Theoretical Physics, University of Innsbruck, Technikerstraße 21a, A-6020 Innsbruck, Austria \\ ${ }^{3}$ NEST and Dipartimento Tecnologie dell'Informazione, Universitá di Milano, via Bramante 65, I-26013 Crema (CR), Italy \\ ${ }^{4}$ Dipartimento di Scienze Fisiche ed Astronomiche, Universitá di Palermo, via Archirafi 36, I-90123 Palermo, Italy \\ ${ }^{5}$ University of Minas Gerais, Belo Orizonte, Brazil \\ ${ }^{6}$ School of Physics and Astronomy, University of Leeds, LS2 9JT, United Kingdom \\ (Received 13 July 2005; published 21 April 2006)
}

\begin{abstract}
We propose a new way to generate an observable geometric phase by means of a completely incoherent phenomenon. We show how to imprint a geometric phase to a system by adiabatically manipulating the environment with which it interacts. As a specific scheme, we analyze a multilevel atom interacting with a broadband squeezed vacuum bosonic bath. As the squeezing parameters are smoothly changed in time along a closed loop, the ground state of the system acquires a geometric phase. We also propose a scheme to measure such a geometric phase by means of a suitable polarization detection.
\end{abstract}

Whenever a pure quantum state undergoes a parallel transport along a closed path, it gathers information on the geometric structure of the Hilbert space in which it lies. In this Letter, we will show that a possible way to generate such a parallel transport is by way of an irreversible quantum evolution. In several models of interaction with the environment, there are some "protected" subspaces, such as the decoherence-free subspaces, which are left unaffected [1]. States lying in these subspaces are stationary; i.e., they do not evolve in time. A typical example is the ground state of an atomic system, which, trivially, remains unaffected by the coupling with the electromagnetic field. However, there are situations in which the interaction between a system and an engineered environment can generate nontrivial ground states [2-6]. For instance, when a group of atoms collectively interacts with a broadband squeezed vacuum, the highly nonclassical correlations which are present in the field are transferred to the atomic system, which relaxes in a complex pure equilibrium state. In such a scenario, the control over the engineered reservoir allows an indirect control on the state of the system to which it is coupled [7]. Of particular interest is the possibility to change in time the reservoir parameters in such a way that the protected system subspace evolves in a controlled fashion. Here we show that, if this change in time is made slowly enough, a state lying in such a subspace evolves coherently and acquires information about the geometry of the space explored.

As an explicit example, we consider a suitable multilevel atomic system interacting with a broadband squeezed vacuum. To be more specific, let us consider first a threelevel atom whose interaction with an electromagnetic field in the rotating wave approximation is described by the following Hamiltonian:

$$
H=H_{S}+\int \hat{a}^{\dagger}(\omega) \hat{a}(\omega) d \omega+\int\left[g(\omega) S^{\dagger} \hat{a}(\omega)+\text { H.c. }\right] d \omega,
$$

where $H_{S}=\Omega \sum_{k=-1}^{1} k|k\rangle\langle k|$ is the free atomic Hamiltonian, $S=|-1\rangle\langle 0|+| 0\rangle\langle 1|$ is the atomic operator describing the absorption of an excitation, and $\hat{a}(\omega)$ is the annihilation operator of the mode with frequency $\omega(\hbar=$ 1). The field, which we treat as a reservoir, is assumed to be in a broadband squeezed vacuum state. In mathematical terms, this is obtained from the ordinary field vacuum state by means of the unitary operator $\hat{K}(\eta)$

$$
|\operatorname{vac}(\eta)\rangle_{\mathrm{sq}}=\hat{K}(\eta)|\mathrm{vac}\rangle,
$$

where

$$
\hat{K}(\eta)=\exp \frac{1}{2}\left\{\int\left[\eta \hat{a}^{\dagger}(\Omega-\omega) \hat{a}^{\dagger}(\Omega+\omega)-\text { H.c. }\right] d \omega\right\}
$$

is a multimode squeezing transformation [1,3], which correlates symmetrical pairs of modes around the carrier frequency $\Omega$, and $\eta=e^{i \varphi} r$ is the squeezing parameter, whose polar coordinates $\varphi \in\{0 \ldots 2 \pi\}$ and $r>0$ are called the phase and amplitude of the squeezing, respectively.

The use of the Born Markov approximation, justified by the broadband nature of the field, leads to the following master equation for the atomic degrees of freedom [1,3]:

$$
\frac{d \rho}{d t}=-\frac{\Gamma}{2}\left\{R^{\dagger} R \rho+\rho R^{\dagger} R-2 R \rho R^{\dagger}\right\},
$$

where $\Gamma=2 \pi|g(\Omega)|^{2}$, and

$$
R(\eta)=S \cosh r+e^{i \varphi} S^{\dagger} \sinh r .
$$

From (4) follows that the state

$$
\left|\psi_{\mathrm{DF}}(\eta)\right\rangle=c|-1\rangle-e^{i \varphi} s|1\rangle,
$$

with $c(r)=\cosh r / \sqrt{\cosh 2 r}$ and $s(r)=\sinh r / \sqrt{\cosh 2 r}$, satisfies $R(\eta)\left|\psi_{\mathrm{DF}}(\eta)\right\rangle=0$. In other words, this state is 
unaffected by the environment; i.e., it is decoherence-free. Moreover, $|\psi(\eta)\rangle$ represents the new ground state, as all the other states of the atomic system relax to it.

As anticipated, the key idea is to smoothly change the squeezing parameter of the field in order to "adiabatically" drag a state initially prepared in $\left|\psi_{\mathrm{DF}}\left(\eta_{0}\right)\right\rangle$ into $\left|\psi_{\mathrm{DF}}\left(\eta_{t}\right)\right\rangle$, where $\eta_{t}$ is the time dependent squeezing parameter. We will show the existence of an "adiabatic" limit such that the transition probability of $\left|\psi_{\mathrm{DF}}(\eta)\right\rangle$ to the orthogonal subspace vanishes as the rate of change of $\eta$ becomes sufficiently small. Furthermore, we will show that, after a cyclic evolution of $\eta$, the state $\left|\psi_{\mathrm{DF}}\right\rangle$ acquires a geometric phase. It is worth stressing that this procedure, although reminiscent of the usual adiabatic evolution, is a different physical phenomenon. The usual adiabatic approximation refers to a coherent evolution, obtained by tuning the parameters of the system Hamiltonian, while the "steering process" discussed here is achieved by manipulating the environment. The essential difference is that in the latter case the system state can be adiabatically controlled entirely by means of an incoherent phenomenon and no Hamiltonian term contributes to its time evolution. To show how this incoherent adiabatic steering process can take place, consider the time dependent version of Eq. (3), where $R\left(\eta_{t}\right)$ is explicitly dependent on time through $\eta_{t}$. It is useful to express the equation of motion in the reference frame where $\psi_{\mathrm{DF}}$ is time independent. To this end, consider the following unitary transformation:

$$
O(\eta)=\left(\begin{array}{ccc}
c(r) e^{-i(\varphi / 2)} & 0 & s(r) e^{i(\varphi / 2)} \\
0 & 1 & 0 \\
-s(r) e^{-i(\varphi / 2)} & 0 & c(r) e^{i(\varphi / 2)}
\end{array}\right),
$$

from the basis $|1\rangle,|0\rangle,|-1\rangle$ to the time dependent basis $|\tilde{1}\rangle$, $|\tilde{0}\rangle,|-\tilde{1}\rangle$, where $\left|\psi_{\mathrm{DF}}\right\rangle$ coincides with $|-\tilde{1}\rangle$ Under this change of frame, the equation of motion becomes

$$
\frac{d \tilde{\rho}}{d t}=-\frac{\tilde{\Gamma}}{2}\left(\tilde{R}^{\dagger} \tilde{R} \tilde{\rho}+\tilde{\rho} \tilde{R}^{\dagger} \tilde{R}-2 \tilde{R} \tilde{\rho} \tilde{R}^{\dagger}\right)-i[G, \tilde{\rho}],
$$

where, in this new frame, $\tilde{\rho}=O\left(\eta_{t}\right) \rho O^{\dagger}\left(\eta_{t}\right), \quad \tilde{R}=$ $O\left(\eta_{t}\right) R\left(\eta_{t}\right) O^{\dagger}\left(\eta_{t}\right) / \sqrt{\cosh 2 r}, \quad \tilde{\Gamma}=\Gamma \cosh 2 r, \quad$ and $G=$ $\mathrm{idO} / \mathrm{dt} \mathrm{O}^{\dagger}$ is a Hamiltonian term arising from the change of picture. Moreover, in this frame the Lindbladian term $\tilde{R}^{\dagger} \tilde{R}$ assumes a simple diagonal form:

$$
\tilde{R}^{\dagger} \tilde{R}=|\tilde{1}\rangle\langle\tilde{1}|+| \tilde{0}\rangle\langle\tilde{0}| .
$$

The main advantage of this transformation is that it allows one to formulate clearly the adiabatic condition, since the rate of change of the environment parameters is contained in the operator $G$. The limit that we are interested in is the one in which the dominant contribution in Eq. (7) comes from the incoherent terms, i.e., $|G| \ll \tilde{\Gamma}$.

An interesting case is the one in which the squeezing amplitude is kept constant while its phase is slowly changed from 0 to $2 \pi$. This adiabatic evolution can be easily achieved by tuning, for example, the carrier frequency $2 \Omega$ of the squeezed state slightly off resonance from the two photon transition $|-1\rangle \leftrightarrow|1\rangle$. By introducing this detuning $\delta$ (assuming $\delta \ll \Omega$ ), the master equation obtained has the form of Eqs. (3) and (4), where $\varphi$ is replaced by $\varphi_{t}=\varphi_{0}+\delta t$. Hence, a sufficiently small value of $\delta$ determines the required adiabatic evolution. Under this condition, the operator $G$ assumes the form

$$
G=\frac{\dot{\varphi}_{t}}{2}\left(\begin{array}{ccc}
\alpha & 0 & \beta \\
0 & 0 & 0 \\
\beta & 0 & -\alpha
\end{array}\right),
$$

where $\alpha=\frac{1}{\cosh 2 r}$ and $\beta=-\frac{\sinh 2 r}{\cosh 2 r}$. We show that, when $\dot{\varphi}$ is small enough, the state $|-1\rangle \equiv\left|\psi_{\mathrm{DF}}\right\rangle$ is adiabatically decoupled from its orthogonal subspace and a cyclic evolution in $\varphi$ results in a geometric phase acquired by $\left|\psi_{\mathrm{DF}}\right\rangle$ depending only, in this case, on the amount of squeezing $r$. Note, however, that, since the steering process is essentially incoherent, any phase information acquired by a superposition of $\psi_{\mathrm{DF}}$ and a state belonging to the orthogonal subspace is inevitably lost, as the latter is subject to decoherence. The only way to retain such information is to consider an auxiliary level $|a\rangle$, unaffected by the noise, playing the role of a reference state for an interferometric measurement. For simplicity, assume that $|a\rangle$ is unaffected by the environment during the whole evolution and, hence, is time independent. As a consequence, the action of the unitary transformation $O$ on $|a\rangle$ is trivial, and Eq. (7) remains essentially unchanged.

The whole information about the geometrical phase and the coherence retained by the system during its evolution is then recorded in the phase and amplitude of the density matrix term $\rho_{-a}=\langle-1|\tilde{\rho}| a\rangle$, whose evolution is described by the following set of coupled differential equations:

$$
\begin{aligned}
\dot{\rho}_{-a} & =-i\langle a|G \rho| \tilde{1}\rangle=i\left(\alpha \rho_{a+}-\beta \rho_{-a}\right) \frac{\dot{\varphi}}{2}, \\
\dot{\rho}_{+a} & =-\frac{\tilde{\Gamma}}{2} \rho_{+a}-i\left\langle\tilde{1}\left|G_{\rho}\right| a\right\rangle \\
& =-\frac{1}{2}(\tilde{\Gamma}+i \alpha \dot{\varphi}) \rho_{+a}-i \beta \rho_{-a} \frac{\dot{\varphi}}{2},
\end{aligned}
$$

where $\rho_{+a}=\langle 1|\tilde{\rho}| a\rangle$. Assume that initially the excited states $|1\rangle$ and $|0\rangle$ of the system are not populated; hence, $\rho_{+a}(0)=0$, and the coherence $\rho_{-a}$ evolves as

$$
\begin{aligned}
\rho_{-a}(t)= & \rho_{-a}(0) \frac{1}{\left(\lambda_{-}-\lambda+\right)}\left[\left(\lambda_{-}+i \alpha \dot{\varphi}\right) e^{\lambda_{+} t}\right. \\
& \left.-\left(\lambda_{+}+i \alpha \dot{\varphi}\right) e^{\lambda_{-} t}\right],
\end{aligned}
$$

where $\lambda_{ \pm}=-(\tilde{\Gamma} / 4) \mp \frac{1}{2} \sqrt{\left(\tilde{\Gamma}^{2} / 4\right)+i \alpha \tilde{\Gamma} \dot{\varphi}-\dot{\varphi}^{2}}$.

In the limit $\Gamma \gg \dot{\varphi}$, we obtain for the coherence $\rho_{-a}$

$$
\begin{aligned}
\rho_{-a}(t)= & \rho_{-a}(0)\left((1-\epsilon) e^{i(\varphi / 2) \dot{\varphi} t-\epsilon \tilde{\Gamma} t}\right. \\
& \left.+\epsilon e^{-i(\varphi / 2) \dot{\varphi} t-(\tilde{\Gamma} / 2)(1-2 \epsilon) t}\right)
\end{aligned}
$$


where $\epsilon \simeq\left(\beta^{2} / 2\right)(\dot{\varphi} / \tilde{\Gamma})^{2}$. We are interested in a cyclic evolution, corresponding to $T=2 \pi / \dot{\varphi}$. By retaining only the leading terms in $\dot{\varphi} / \Gamma$, the total evolution at time $T$ is given by

$$
\rho_{-a}(T)=\rho_{-a}(0) e^{i \pi \alpha-\alpha \beta^{2} \pi(\dot{\varphi} / \Gamma)},
$$

where we have substituted $\tilde{\Gamma}$ with $\Gamma / \alpha$. Finally, going back to the original frame by means of $O^{\dagger}(T)$, the corresponding coherence $\rho_{\psi a}(t)=\left\langle\psi_{\mathrm{DF}}|\rho(t)| a\right\rangle$ is given by:

$$
\rho_{\psi a}(T)=\rho_{\psi a}(0) e^{-i \pi(1-\alpha)-\alpha \beta^{2} \pi(\dot{\varphi} / \Gamma)} .
$$

For example, a state initially prepared in $|\tilde{\psi}(0)\rangle=$ $(1 / \sqrt{2})\left(\left|\psi_{\mathrm{DF}}(0)\right\rangle+|a\rangle\right)$, after closing the loop, evolves into

$$
\begin{aligned}
\rho(T)= & \left.\left.\left(1-\alpha \beta^{2} \pi \frac{\dot{\varphi}}{\Gamma}\right) \mid \tilde{\psi}(T)\right)\right\rangle\langle\tilde{\psi}(T)| \\
& +\alpha \beta^{2} \pi \frac{\dot{\varphi}}{\tilde{\Gamma}}\left|\psi_{\mathrm{DF}}\right\rangle\left\langle\psi_{\mathrm{DF}}\right|,
\end{aligned}
$$

where $|\tilde{\psi}(T)\rangle=(1 / \sqrt{2})\left(e^{-i \pi(1-\alpha)}\left|\psi_{\mathrm{DF}}(0)\right\rangle+|a\rangle\right)$.

It is clear from this expression that, in the limit $\xi=$ $\dot{\varphi} / \Gamma \ll 1$, the dominant contribution to the time evolution is just a phase factor $e^{i \phi}$, with $\phi=-\pi(1-\alpha)$. This proves that, in the adiabatic approximation, the system preserves its coherence. In fact, according to Eq. (12), the amplitude damping of $\rho_{\psi a}$ occurs only when we take into account the first order contribution in $\xi$, which shows an exponential decay rate of the order of $\beta^{2}(\dot{\varphi} / \Gamma)$. This proves that for small $\dot{\varphi}$ the system admits an adiabatic limit, in which the subspace $H_{\mathrm{DF}}(t)$ spanned by $\left|\psi_{\mathrm{DF}}(t)\right\rangle$ and $|a\rangle$ is adiabatically decoupled from its orthogonal subspace $H_{\perp}(t)$. For this reason, $H_{\mathrm{DF}}(t)$ is decoupled from the effects of the decoherence, which affect only states lying in its orthogonal subspace.

Within this approximation, then, a state prepared in the space $H_{\mathrm{DF}}(0)$ is adiabatically transported rigidly inside the evolving subspace $H_{\mathrm{DF}}(t)$. As a result of this adiabatic steering, when the system is brought back to its initial configuration, the coherence $\rho_{\psi a}$ acquires a phase $\phi=$ $-\pi(1-\alpha)$. This phase can be interpreted as the geometric phase accumulated by the state $\left|\psi_{\mathrm{DF}}(t)\right\rangle$. By using the canonical formula for the Berry phase, it easy to see that the geometric phase of $\left|\psi_{\mathrm{DF}}(t)\right\rangle$ is given by

$$
\begin{aligned}
\chi_{g} & =i \oint\left\langle\psi_{\mathrm{DF}}|d| \psi_{\mathrm{DF}}\right\rangle=i \int_{0}^{2 \pi}\left\langle\psi_{\mathrm{DF}}\left|\frac{d}{d \varphi}\right| \psi_{\mathrm{DF}}\right\rangle d \varphi \\
& =-\pi(1-\alpha)=\phi .
\end{aligned}
$$

As expected, the value of $\phi$ depends only on the squeezing and vanishes as the squeezing tends to zero. Moreover, notice that the phase $\phi$ is purely geometrical; i.e., there is no dynamical contribution arising from an existing Hamiltonian, since, in the absence of any steering process, the states inside $H_{\mathrm{DF}}$ have a trivial dynamics. This makes the measurement of this phase a relatively easy task. Usual procedures to measure geometric phases make use of suitably designed techniques to eliminate dynamical phase contributions, such as spin-echo [8] or parallel transport conditions [9]. In this setup, the geometric phase is the only contribution to the phase accumulated by $\left|\psi_{\mathrm{DF}}\right\rangle$, and, hence, it is straightforward to measure by a suitable interferometric setup.

A simple scheme to measure the geometric phase obtained by such a steering process can be realized with a simple variation of our system. Let us consider the fivelevel atomic system shown in Fig. 1(b). It essentially consists of two replicas of the three-level system discussed above, with the level $|0\rangle$ in common. The important ingredient is that transitions $|0\rangle \leftrightarrow|1\rangle$ and $|-1\rangle \leftrightarrow|0\rangle$ are coupled with modes of the reservoir which are different from those coupled to the transitions $|0\rangle \leftrightarrow\left|1^{\prime}\right\rangle$ and $\left|-1^{\prime}\right\rangle \leftrightarrow|0\rangle$. A simple way to achieve this is to choose, for example, polarization selective transitions, say, leftcircularly polarized modes for the former transitions and right-circularly polarized for the latter ones. The complete Hamiltonian of such a system is

$$
\begin{aligned}
H= & H_{S}+\sum_{i=1,2} \int \hat{a}_{i}^{\dagger}(\omega) \hat{a}_{i}(\omega) d \omega \\
& +\sum_{i=1,2} \int\left[g_{i}(\omega) S_{i}^{\dagger} \hat{a}_{i}(\omega)+\text { H.c. }\right] d \omega,
\end{aligned}
$$

where $H_{S}=\Omega \sum_{k=-1}^{1} k\left(|k\rangle\left\langle k|+| k^{\prime}\right\rangle\left\langle k^{\prime}\right|\right), S_{1}=|-1\rangle\langle 0|+$ $|0\rangle\langle 1|$ and $S_{2}=\left|-1^{\prime}\right\rangle\langle 0|+| 0\rangle\left\langle 1^{\prime}\right|$, and $\hat{a}_{i}(\omega)$ is the annihilation operator of the mode with the energy $\omega$ and polarization $i \in\{1,2\}$. Assume broadband squeezed vacuum states for the set of modes $\hat{a}_{1}(\omega)$ and modes $\hat{a}_{2}(\omega)$ with different squeezing parameters $\eta_{1}=r_{1} e^{i \varphi_{1}}$ and $\eta_{2}=$ $r_{2} e^{i \varphi_{2}}$ :

$$
\left|\operatorname{vac}\left(\eta_{1}, \eta_{2}\right)\right\rangle_{\mathrm{sq}}=\hat{K}_{1}\left(\eta_{1}\right) \hat{K}_{2}\left(\eta_{2}\right)|\mathrm{vac}\rangle,
$$

where $\hat{K}_{i}\left(\eta_{i}\right)$ are the analogs of the operator (2) acting on (a)

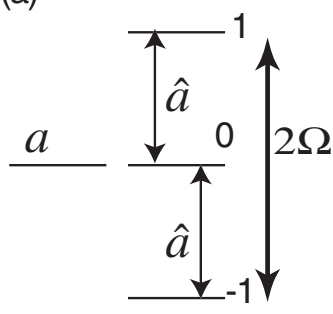

(b)

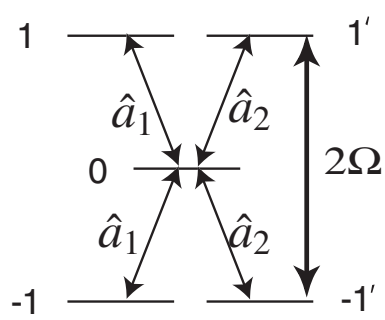

FIG. 1. (a) Schematic representation of the four systems considered. The energy gap between states $|-1\rangle$ and $|0\rangle$ and between $|0\rangle$ and $|1\rangle$ is $\Omega$. The transitions between these levels are coupled to the modes $\hat{a}(\omega)$ of the reservoir. The reference state $|a\rangle$ is decoupled from the reservoir. (b) Five-level system, transitions $1 \leftrightarrow 0$ and $0 \leftrightarrow-1$ are coupled to modes $\hat{a}_{1}(\omega)$ and $1^{\prime} \leftrightarrow 0$ and $0 \leftrightarrow-1^{\prime}$ are coupled to modes $\hat{a}_{2}(\omega)$ of the reservoir. 
the set of modes $\hat{a}_{i}$. Under the same assumptions which lead to Eq. (3), we obtain the master equation:

$$
\frac{d \rho}{d t}=-\sum_{i} \frac{\Gamma_{i}}{2}\left\{R_{i}^{\dagger} R_{i} \rho+\rho R_{i}^{\dagger} R_{i}-2 R_{i} \rho R_{i}^{\dagger}\right\},
$$

where $\quad \Gamma_{i}=2 \pi\left|g_{i}(\Omega)\right|^{2} \quad$ and $\quad R_{i}\left(\eta_{i}\right)=S_{i} \cosh r_{i}+$ $e^{i \varphi_{i}} S_{i}^{\dagger} \sinh r_{i}$. This system admits a two-dimensional decoherent-free subspace, spanned by states $\left|\psi_{1}\right\rangle$ and $\left|\psi_{2}\right\rangle$ whose definition is the analog of state $\left|\psi_{\mathrm{DF}}\right\rangle$ of Eq. (5). We assume again time dependent squeezing parameters $\eta_{i}^{t}$, and again we examine the time dependence of the system in a rotating frame, i.e., a frame where the state $\left|\psi_{i}(t)\right\rangle$ appears stationary. This leads to the following master equation for the five-level system in the rotating frame:

$$
\begin{aligned}
\frac{d \tilde{\rho}}{d t}= & -\sum_{i} \frac{\tilde{\Gamma}_{i}}{2}\left(\tilde{R}_{i}^{\dagger} \tilde{R}_{i} \tilde{\rho}+\tilde{\rho} \tilde{R}_{i}^{\dagger} \tilde{R}_{i}-2 \tilde{R}_{i} \tilde{\rho} \tilde{R}_{i}^{\dagger}\right) \\
& -i \sum_{i}\left[G_{i}, \tilde{\rho}\right]
\end{aligned}
$$

where $G_{i}=i d O / d \eta_{i} O^{\dagger} \dot{\eta}_{i}, O(t)$ being the unitary transformation producing the change of frame. Assume again, for simplicity, that the parameters $r_{1}$ and $r_{2}$ are kept constant and that $\varphi_{1}=\varphi_{2}=\varphi$. Under this assumption, the master equation can be exactly solved. The solution is analogous to the one obtained for the system previously analyzed. Suppose that the system is initially prepared in a coherent superposition of state $\left|\psi_{1}\left(\eta_{1}^{0}\right)\right\rangle$ and $\left|\psi_{2}\left(\eta_{2}^{0}\right)\right\rangle$, for example: $|\psi(0)\rangle=(1 / \sqrt{2})\left(\left|\psi_{1}\left(\eta_{1}^{0}\right)\right\rangle+\left|\psi_{2}\left(\eta_{2}^{0}\right)\right\rangle\right)$. At a later time, one has

$$
\rho_{\psi_{1} \psi_{2}}(t)=\frac{1}{2} \exp \left\{-i\left(\alpha_{2}-\alpha_{1}\right) \frac{\dot{\varphi}}{2}-\left(\frac{\beta_{1}^{2}}{2 \tilde{\Gamma}_{1}}+\frac{\beta_{2}^{\prime 2}}{2 \tilde{\Gamma}_{2}}\right) \dot{\varphi}^{2}\right\} t,
$$

with $\alpha_{i}=1 / \cosh 2 r_{i}$ and $\beta_{i}=-\left(\sinh 2 r_{i} / \cosh 2 r_{i}\right)$. When the parameter $\varphi$ closes a loop, at $t=T=2 \pi / \dot{\varphi}$, the coherence has gained a phase

$$
\chi=\pi i\left(\alpha_{2}-\alpha_{1}\right)=\phi_{2}-\phi_{1},
$$

which is the difference between the geometric phases $\phi_{i}=$ $\pi\left(1-\alpha_{i}\right)$ acquired by the states $\left|\psi_{i}\right\rangle$, respectively. As in the previous scheme, the visibility is reduced by a factor which is linear in the "adiabatic parameters" $\dot{\varphi} / \Gamma_{i}$, which guarantees the existence of the adiabatic limit. The advantage of this modified scheme is that the value of the geometric phases can be readily measured from the polarization of the light emitted when the system relaxes. In fact, if the value of the squeezing parameters $r_{i}$ is switched suddenly to zero, the states $\left|\psi_{i}\right\rangle$ are no longer decoherencefree and decay to a superposition of the ground states $|-1\rangle$ and $\left|-1^{\prime}\right\rangle$. This dissipation process is accompanied by two photon emissions into the reservoir. Because of the structure of the interaction (14) with the reservoir, the photon emitted due to the transitions $|1\rangle \rightarrow|0\rangle$ and $\left|1^{\prime}\right\rangle \rightarrow|0\rangle$ is polarized according to the geometric phase accumulated between $\left|\psi_{1}\right\rangle$ and $\left|\psi_{2}\right\rangle$. For example, if $\hat{a}_{1}(\omega)$ and $\hat{a}_{2}(\omega)$ are right- and left-circularly polarized modes, respectively, the first dissipation process produces the linearly polarized photon:

$$
\left|\psi_{1}\right\rangle+e^{i\left(\varphi_{1}-\varphi_{2}\right)}\left|\psi_{2}\right\rangle \rightarrow|R\rangle+e^{i\left(\varphi_{1}-\varphi_{2}\right)}|L\rangle .
$$

The detection of the polarization of the emitted photon makes possible a direct measurement of the geometric phase.

We have presented a scheme to generate a geometric phase via a completely incoherent control procedure. This scheme is conceptually different from the usual coherent adiabatic control. The latter is realized through a smooth evolution of suitable Hamiltonians, whereas here the adiabatic steering is the effect of an externally controlled environment. The phase generated is purely geometrical and, therefore, experimentally detectable without resorting to techniques for the elimination of dynamical contributions. Because of its very nature, this scheme is immune from unwanted environmental effects. Moreover, like any geometric effects, it presents an inherent degree of robustness against uncertainties in the control parameters.

This work was supported in part by the EU under grant IST-TOPQIP, "Topological Quantum Information Processing" (Contract No. IST-2001-39215). V. V. also acknowledges support from EPSRC and the British Council in Austria. A.C. acknowledges support from Marie Curie RNT Project CONQUEST.

[1] G. M. Palma, K.-A. Suominen, and A. K. Ekert, Proc. R. Soc. A 452, 567 (1996); L.-M. Duan and G.-C. Guo, Phys. Rev. Lett. 79, 1953 (1997); P. Zanardi and M. Rasetti, Phys. Rev. Lett. 79, 3306 (1997); D. A. Lidar, I. L. Chuang, and K.B. Whaley, Phys. Rev. Lett. 81, 2594 (1998); A. Barenco et al., SIAM J. Comput. 26, 1541 (1997).

[2] A. K. Ekert, G. M. Palma, S. Barnett, and P. L. Knight, Phys. Rev. A 39, 6026 (1989).

[3] G. S. Agarwal and R. R. Puri, Phys. Rev. A 41, 3782 (1990).

[4] J. F. Poyatos, J. I. Cirac, and P. Zoller, Phys. Rev. Lett. 77, 4728 (1996).

[5] C. J. Myatt et al., Nature (London) 403, 269 (2000).

[6] A. Kuzmich, K. Mølmer, and E. S. Polzik, Phys. Rev. Lett. 79, 4782 (1997).

[7] A. R. R. Carvalho, P. Milman, R. L. de Matos Filho, and L. Davidovich, Phys. Rev. Lett. 86, 4988 (2001).

[8] J. A. Jones, V. Vedral, A. Ekert, and G. Castagnoli, Nature (London) 403, 869 (2000); A. Ekert et al., J. Mod. Opt. 47, 2501 (2000).

[9] Y. Aharonov and J. Anandan, Phys. Rev. Lett. 58, 1593 (1987). 